\title{
An extension of the time ratio theory of sedimentation tanks
}

\author{
G. A. PRICE
}

Mr W. H. Emery, University of Sheffield

I am carrying out extensive tests to further the work of Clements, ${ }^{1}$ Clements and Khattab ${ }^{13}$ and the Author in the field of sedimentation tank design. A study is being made of restrained wall jet expansions and lateral mixing with a view to their effect on the time ratio theory and prediction.

53. The time ratio proposed by Clements, ${ }^{1}$ developed by Khattab, ${ }^{5}$ and extended by the Author to include longitudinal mixing, assumes the circulating eddy to be ineffective. In $\S 28$ the Author assumes 'no particles are transferred into the ineffective part of the tank', i.e. the circulating eddy. This is in effect assuming that there is no lateral mixing. Recent experiments conducted at the University of Sheffield have produced results which disagree with this assumption.

54. Longitudinal and lateral mixing are generally assumed to depend on the state of turbulence and mean velocity gradients at the point in question. The inlet zone of a sedimentation tank will always be the zone of maximum kinetic energy and hence the maximum state of turbulence and mean velocity gradients. It is therefore reasonable to assume that the maximum mixing, both lateral and longitudinal, will occur at the inlet zone and reduce down the tank; the actual reduction will depend on the inlet arrangements.

55. In these experiments and in reference 4 it has been noted that the maximum lateral velocity gradients are significantly greater than the maximum longitudinal velocity gradients. Also the maximum lateral velocity gradient, if the boundary layer is ignored, occurs at the interface of the circulating eddy and the through flow jet, and not as illustrated in Fig. 2 (Fig. 8).

56. From the work of Hazen ${ }^{3}$ and Clements, ${ }^{1}$ who both show that vertical circulating eddies do not reduce the tank efficiency if scour is avoided, it seems that lateral mixing will be significant only in horizontal circulating eddies. The effect of this mixing will be to increase the removal in the tank due to an increased effective surface area, this being greatest in tanks with a low time ratio valve. Support for this view is obtained from Thompson' ${ }^{14}$ observations that there was no visible difference in settlement over sedimentation tank floors.

Mr M. S. Clements, University of Sheffield

The simple theory published in $1966^{1}$ took no account of mixing and the Author has shown how longitudinal mixing can be allowed for by following up Hazen's approach ${ }^{3}$ in the case of an ideal tank.

58. Some research at the University of Sheffield is aimed at testing the statement made in $\S 50$. Under a Science Research Council research programme about twenty 


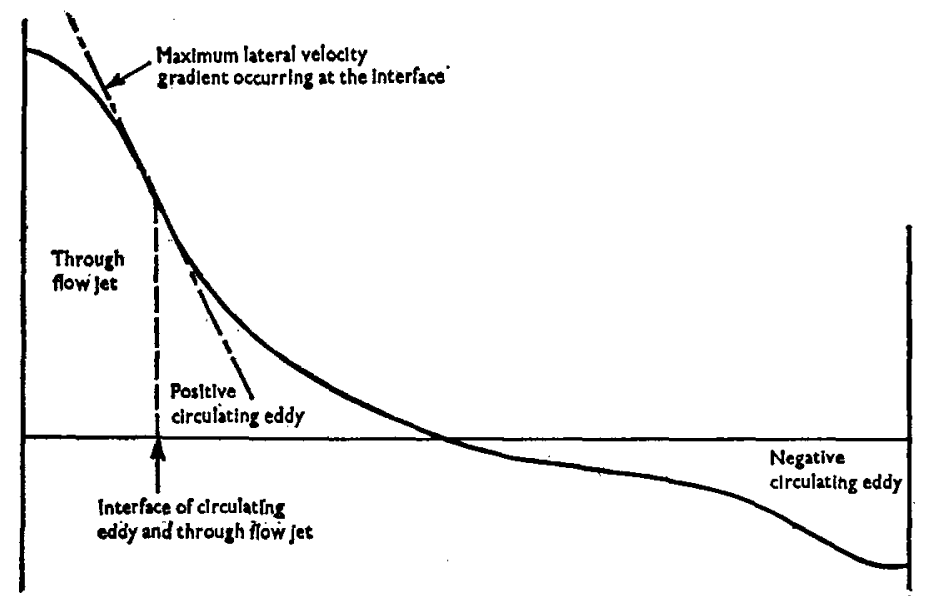

Fig. 8. Velocity variation over breadth (typical experimental result)

tanks, settling primary sludge, secondary sludge, humus and activated sludge are being examined and the removals at known loadings are being compared with the results of static settling column tests which give information on the settling characteristics of the particles. In making the comparisons many variables have been taken into account (e.g. slight differences in depth between tank and column, and slight temperature differences). Although the work is not complete the time ratio method, when used without a correction for longitudinal mixing, is consistently forecasting (on the basis of static settling column results) removals a few per cent lower than are actually achieved in the tank. It was to be expected that tank turbulence would give rise to more flocculation, and therefore greater removals, than take place in a static column. This effect and that of lateral mixing are therefore seen to be greater than the effect of longitudinal mixing in the cases examined and are particularly noticeable in tanks with low time ratios. In these cases the effect of longitudinal mixing is very small (as the Author points out in $\S 37$ ) and one can expect flocculation and lateral mixing effects to be greater than for high time ratios.

59. The use of static settling column results and the time ratio theory in its simplest form (i.e. the proportion of effective tank surface area being taken as equal to the time ratio) therefore appear to give an underestimate of the removal achieved. In the interests of economy and good design the work is continuing in order to give a more precise basis; it is hoped to assess quantitatively the separate effects of longitudinal and lateral mixing and of flocculation.

60. However, in view of the variable nature of sewage which is often found from day to day even at one works, one wonders whether, with greater precision available, the designer will need to design tanks for the worst possible case or whether a more realistic quality control approach should now be adopted, with perhaps $95 \%$ of samples being required to have standards better than specified figures.

\section{Dr Price}

In reply to Mr Emery's comments regarding § 28, the basis for the assumption was that suspended solids would be transferred both into and out of the circulating eddy along the mixing face; in the absence of experimental data to prove otherwise the net summation of solids transfer was assumed to equate to zero. 
62. I accept that particles will mix into the circulating eddy and settle out; however, the converse mechanism of particles leaving the eddy region could result in reduced settling efficiency depending on the particle height at transfer and the velocity distributions in the flow through jet at the point of re-entry and downstream sections.

63. It is agreed that maximum longitudinal and lateral mixing will occur in the inlet zone but in my experience it is unlikely that eddy velocities in this region would allow settling without scouring to take place. Towards the outlet eddy velocities may be sufficiently reduced to allow settling, but because mixing will also be reduced due to smaller velocity gradients the increase in effective surface area due to lateral mixing may well be small. Mr Emery does not quantify his experimental results although Mr Clements mentions that one aim of the current research is to enable the effects of both longitudinal and lateral mixing and flocculation to be quantified.

64. The theory in the Paper considers the worst case effect of perfect longitudinal mixing on discrete particles. Current research indicates that other factors have equal if not more significance on settling efficiency and so it would be appropriate if coefficients accounting for each variable could be incorporated into the relationships in \$49. The feeling obtained is that the findings of the current research will give a greater understanding of the relative importance of the many design parameters and such information will be much applauded in the design office.

65. Mr Clements points out the difficulties of designing tanks to treat the variable nature of sewage arriving at one works. As certain sewage characteristics will be such that settling efficiency can be increased by promoting flocculation, the accepted design principle of uniform velocity throughout the tank may not be the optimum. For these sewages and where facilities for pre-mixing and addition of coagulants to aid settling are not available, a variable inlet arrangement, e.g. removable baffle, that would encourage mixing in the inlet zone when so desired is one answer. To avoid operational difficulties with such an inlet, however, quality control as suggested by $\mathrm{Mr}$ Clements may be the most practical means of dealing with this complex problem.

\section{References}

13. Clements M. S. and Khattab A. F. M. Time ratio in radial flow sedimentation tanks. Proc. Instn Civ. Engrs, 1968, 40, Aug., 471-494.

14. Thомpson D. M. Discussion on Scaling laws for continuous flow sedimentation in rectangular tanks. Proc. Instn Civ. Engrs, 1970, 46, July, 390-393. 